\title{
Molecular Model of a Quantum Dot Beyond the Constant Interaction Approximation
}

\author{
Ruslan Temirov, ${ }^{1,2, *}$ Matthew F. B. Green, ${ }^{1,2}$ Niklas Friedrich, ${ }^{1,2}$ Philipp Leinen, ${ }^{1,2}$ Taner Esat, ${ }^{1,2}$ Pawel Chmielniak, $, 3,4$ \\ Sidra Sarwar, ${ }^{3,4,2}$ Jeff Rawson, ${ }^{3,4,2}$ Paul Kögerler, ${ }^{3,4,2}$ Christian Wagner, ${ }^{1,2}$ Michael Rohlfing, ${ }^{5}$ and F. Stefan Tautz ${ }^{1,2}$ \\ ${ }^{1}$ Peter Grünberg Institut (PGI-3), Forschungszentrum Jülich, 52425 Jülich, Germany \\ ${ }^{2}$ Jülich Aachen Research Alliance (JARA)-Fundamentals of Future Information Technology, 52425 Jülich, Germany \\ ${ }^{3}$ Institute of Inorganic Chemistry, RWTH Aachen University, 52074 Aachen, Germany \\ ${ }^{4}$ Peter Grünberg Institut (PGI-6), Forschungszentrum Jülich, 52425 Jülich, Germany \\ ${ }^{5}$ Institut für Festkörpertheorie, Westfälische Wilhelms-Universität Münster, 48149 Münster, Germany
}

(Received 27 December 2017; published 18 May 2018)

\begin{abstract}
We present a physically intuitive model of molecular quantum dots beyond the constant interaction approximation. It accurately describes their charging behavior and allows the extraction of important molecular properties that are otherwise experimentally inaccessible. The model is applied to data recorded with a noncontact atomic force microscope on three different molecules that act as a quantum dot when attached to the microscope tip. The results are in excellent agreement with first-principles simulations.
\end{abstract}

DOI: 10.1103/PhysRevLett.120.206801

When a single electron moves into a quantum dot (QD), it must overcome the charging energy $e^{2} / C$, where $C$ is the capacitance of the QD [1,2]. At low temperatures $\left(k_{b} T \ll e^{2} / C\right)$, the occupancy of the QD can be controlled with single-electron precision, presenting a range of promising functionalities [3]. QDs consisting of single atoms or molecules are of particular interest because their charging behavior can be tailored by the chemistry of their constituents ("chemically designed QDs"). This design freedom, however, comes at a price: The constant interaction approximation, which assumes that $e^{2} / C$ is independent of the number $N$ of electrons already present in the quantum dot, is not valid for atomically small QDs $[2,4]$. A quantitative description of single-atom or singlemolecule QDs is therefore challenging. The fundamental reason underlying this difficulty is the fact that the capacitances, in terms of which the charging is conveniently described, cease to be invariants [5,6], and moreover, the effect of $N$ on these collective quantities is difficult to generalize. In this situation, a description of the QD in terms of molecular properties would be desirable.

In this Letter, we present a model that correctly and quantitatively describes the charging physics of nanoscale QDs beyond the constant interaction approximation. Our model provides a straightforward characterization of several single-molecule QDs, allowing the determination of generic properties of the molecules in the QD junction (energy level alignment at the electrodes, intraorbital Coulomb repulsion, and charge state dependence of the polarizability), which are otherwise difficult to access experimentally. We anticipate that the model and the results presented here pave the way towards the deliberate design of molecular QDs with desired properties.
An equivalent circuit of a single-molecule QD junction is shown in Fig. 1(a) [1]: The QD is placed between two leads and electrons are transferred between the upper lead and the QD using the bottom lead as a capacitive gate. Recent progress in noncontact atomic force microscopy (NC-AFM) allows for the controlled fabrication of single-molecule quantum dot junctions of known geometry [7-10]. Our experimental realization of the circuit in Fig. 1(a) is shown in Fig. 1(b) [8,9]. A single molecule [see the inset of Fig. 2(b)], initially adsorbed on a clean $\operatorname{Ag}(111)$ surface, is lifted by a combined CREATEC qPlus tuning fork [11] noncontact atomic force, scanning tunneling microscope (NC-AFM/STM), operated in an ultrahigh vacuum at $5 \mathrm{~K}$. The small qPlus oscillation amplitude of $0.1 \AA$ allows the controlled manipulation of the molecule.

The molecule acts as a QD because its states hybridize weakly with those of the tip electrode. The geometry of the QD junction is also very well controlled: The tunnel junction between the molecular QD and the tip consists of a single chemical bond of length $d \simeq 2.2 \AA$ [13] between the outermost silver atom of the tip apex and a carboxylic oxygen atom of the molecule, while the distance $z$ between the junction's leads (tip and surface) can be readily determined from the force interaction of the lower end of the molecule with the surface and the known length of the molecule $[9,14,15]$. We note in passing that this arrangement has been used to image the electrostatic potentials of nanostructures by scanning quantum dot microscopy (SQDM) $[8,9]$.

Within the canonical single electron box model, the Helmholtz free energy of the QD in the circuit sketched in Fig. 1(a) is 
(a)

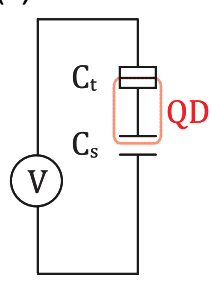

(b)

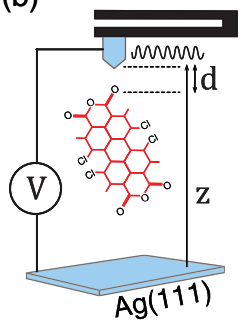

(d)

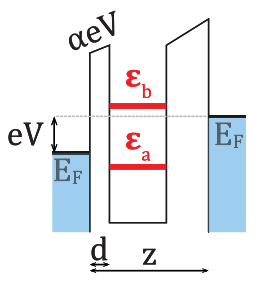

(c)

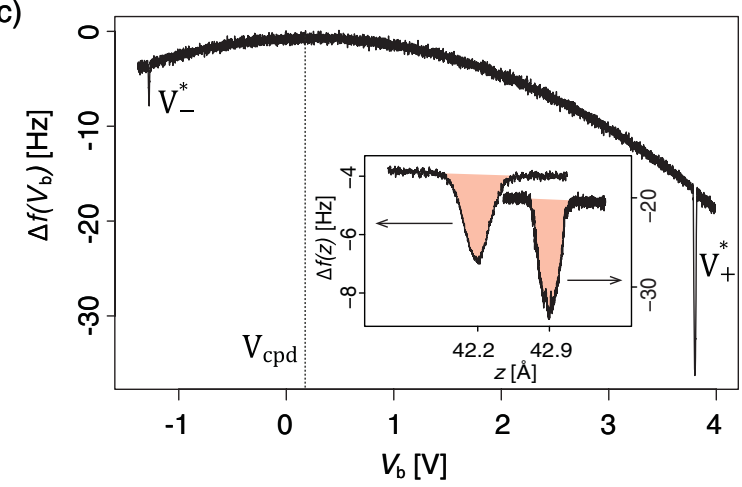

FIG. 1. Structure and charging of the single-molecule QD junction investigated in this work. (a) Equivalent circuit of the QD junction. $C_{t}$ and $C_{s}$ denote capacitances of the tip-molecule and molecule-surface junctions. (b) Experimental realization of the QD junction with PTCDA-Cl ${ }_{4}$. Electron tunneling occurs through the tip-molecule junction, characterized by the distance d. $z$ is the total tip-surface distance. (c) $\Delta f\left(V_{b}\right)$ spectrum recorded at $z=30 \AA$. Two charging events are observed: At $V_{-}^{*}$, one electron is withdrawn from the QD, while at $V_{+}^{*}$, one electron is placed into it. $V_{\mathrm{cpd}}$ is the contact potential difference [12]. The inset shows examples of two experimentally measured $\Delta f(z)$ curves used to calculate $\Delta F_{-}(z=42.2 \AA)$ (left curve, $V_{b}=-1.57 \mathrm{~V}$ ) and $\Delta F_{+}\left(z=42.9 \AA\right.$ ) (right curve, $V_{b}=$ $4.51 \mathrm{~V}$ ) by integration (shaded area). (d) Energy diagram of the QD. Since only two charging voltages $\left(V_{ \pm}^{*}\right)$ are observed, two QD levels can be charged. These levels are shown in red as $\varepsilon_{a}$ and $\varepsilon_{b}$. When a bias voltage $V$ is applied to the junction, both levels are gated by $\alpha e V$ with respect to $E_{F}$ of the left electrode (tip). $V_{-}^{*}$ aligns $\varepsilon_{a}$ with $E_{F}$ of the tip, and one electron is transferred from $\varepsilon_{a}$ to the tip $\left(n_{a}=n_{b}=0\right.$ for $\left.V_{b}<V_{-}^{*}\right) . V_{+}^{*}$ aligns $\varepsilon_{b}$ with $E_{F}$ of the tip, and one electron is transferred from the tip to $\varepsilon_{b}$ $\left(n_{a}=n_{b}=1\right.$ for $\left.V_{b}>V_{+}^{*}\right)$. For $V_{-}^{*}<V_{b}<V_{+}^{*}$, as shown in the Figure, $n_{a}=1, n_{b}=0$.

$A_{\mathrm{SEB}}(V, N)=\sum_{n=1}^{N} E_{n}+\frac{N^{2} e^{2}}{2 C_{\Sigma}}-\frac{C_{s}}{C_{\Sigma}} N e V-\frac{C_{t} C_{s}}{2 C_{\Sigma}} V^{2}$,

where the first term is the sum over the occupied energy levels of the QD. $C_{s}$ and $C_{t}\left(C_{\Sigma} \equiv C_{s}+C_{t}\right)$ are the mutual capacitances of the QD with respect to the two electrodes $[16,17]$. The voltages $V_{ \pm}$at which the electron occupancy of the QD changes from $N$ to $N \pm 1$ are obtained by solving

$$
A\left(V_{ \pm}, N\right)=A\left(V_{ \pm}, N \pm 1\right) .
$$

$V_{ \pm}$can be measured by the NC-AFM, as abrupt changes of the force acting between the QD and the sample [8] (or the

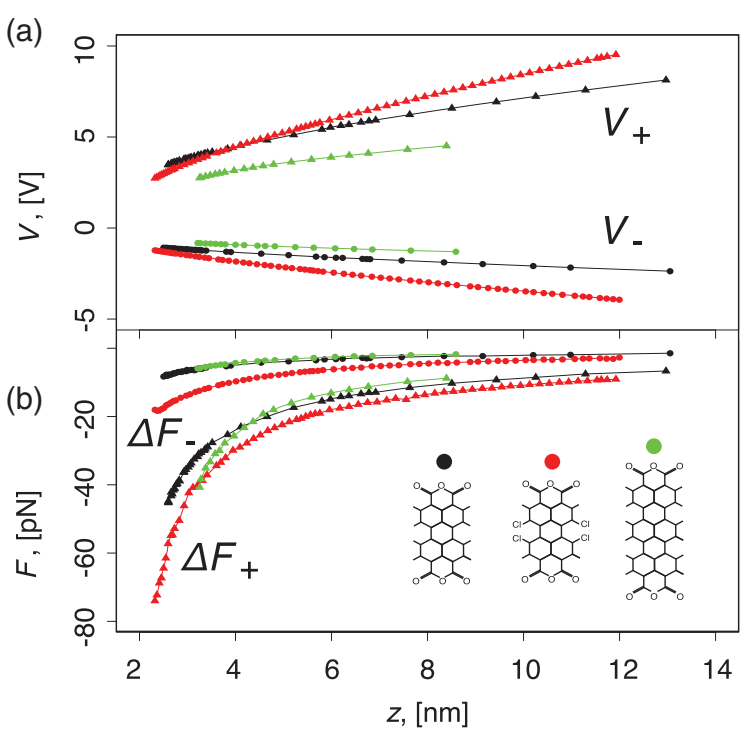

FIG. 2. Experimental characterization of QD junctions with PTCDA (black), PTCDA-Cl ${ }_{4}$ (red), and TTCDA (green). (a) $z$ dependence of the charging voltages $V_{-}$(circles) and $V_{+}$ (triangles). Chemical structures of 3,4,9,10-perylene tetracarboxylic acid dianhydride (PTCDA), 1,6,7,12-tetrachloroperylene3,4,9,10-tetracarboxylic dianhydride (PTCDA-Cl $)$, and 3,4,11,12- terrylene-tetracarboxylic dianhydride (TTCDA) are shown in the inset. (b) $z$ dependence of $\Delta F_{-}$(circles) and $\Delta F_{+}$ (triangles).

tip in a different set-up $[7,10,16-18])$ that occur when the QD changes its occupancy from $N$ to $N \pm 1$. According to Eq. (1), in going from $N$ to $N \pm 1$ the force changes by

$$
\Delta F_{ \pm}=-\left[\partial_{z} A\left(V_{ \pm}, N \pm 1\right)-\partial_{z} A\left(V_{ \pm}, N\right)\right] .
$$

The NC-AFM offers the advantage that not only $V_{ \pm}$but also $\Delta F_{ \pm}$can be measured. $\Delta F_{ \pm}$are obtained by integrating the charging dips of the $\Delta f(z)$ spectra measured at fixed voltages [10] after subtracting a linear background [see inset of Fig. 1(c)]. The background subtraction safeguards that only the force change due to charging contributes to the integrated $\Delta F_{ \pm}$values, and not any change in long-range background forces. Moreover, the oscillation amplitude does not affect the integrated values appreciably [7].

From the four experimental quantities $V_{ \pm}, \Delta F_{ \pm}$, the parameters $E_{N}, E_{N+1}, C_{s}$, and $C_{t}$ of the model in Eq. (1) can thus be calculated using Eqs. (2) and (3). However, as our results show, there is no internally consistent solution. Rather, the two charging events require a different ratio $C_{s} / C_{t}$ [19] as a consequence of a breakdown in the constant interaction approximation due to deformations of the QD confining potential in response to changes in occupancy (nonrigid QD) [4,20].

In order to go beyond the constant interaction approximation the capacitances $C_{s}$ and $C_{t}$ in Eq. (1) must be $N$ dependent. This increases the number of model parameters, 
making their unconstrained identification impossible. At the same time, generating any helpful constraints for $C_{s}(N)$ and $C_{t}(N)$ values is difficult as it requires the application of an intrinsically macroscopic concept, electrostatic capacitance, to a single molecule. As we will show, a description in terms of the molecular polarizability and its change with charge state is a good alternative.

First we present the experimental data. The complete data set $V_{ \pm}(z)$ and $\Delta F_{ \pm}(z)$ for the molecules PTCDA, PTCDA-Cl ${ }_{4}$, and TTCDA is shown in Fig. 2. The successful fabrication of comparable QD junctions from all three molecules is confirmed by the occurrence of two sharp spikes in the $\Delta f\left(V_{b}\right)$ spectrum of each [Fig. 1(c) for PTCDA-Cl $l_{4}$ [8,9]. The $V_{ \pm}$in Fig. 2(a) are the positions of the spikes in $\Delta f\left(V_{b}\right)$ after subtraction of the contact potential difference $V_{\text {cpd }}, V \equiv V_{b}-V_{\text {cpd }}$, and $V_{ \pm} \equiv V_{ \pm}^{*}-$ $V_{\text {cpd }}$ [see Fig. 1(c)]. $V_{ \pm}$are sensitive to the tip-sample distance $z$, as the application of larger voltages is necessary to achieve charging at a larger $z$. Since the frequency shift is related to the vertical force gradient, $\Delta f \approx-\left(d F_{z} / d z\right) \times$ $\left(f_{0} / 2 k_{0}\right)$ [11], an integration $\int_{z-\epsilon}^{z+\epsilon} \Delta f(V, z) d z$ at $V=V_{ \pm}(z)$ [see inset in Fig. 1(c)] yields the force step $\Delta F_{ \pm}$that occurs on charging the QD and that is plotted in Fig. 2(b). While the results displayed in Fig. 2 are similar for the three molecules, there are characteristic differences which, as the molecular model of QD charging will show, are due to differences in the chemical structure of the molecular QDs.

We start the formulation of the molecular QD model by observing that in the constant interaction approximation, the last term of Eq. (1) is independent of $N$, and hence, it cancels in Eqs. (2)-(3). The remaining three terms can be equivalently expressed as $\varepsilon(N)-\alpha N e V$, where $\varepsilon(N)=0$, $\varepsilon_{a}$ or $\varepsilon_{a}+\varepsilon_{b}$ (for $N=0,1,2$ ) is the occupation energy of the QD [Fig. 1(d)], and $\alpha \equiv d \Phi_{\mathrm{QD}} / d V$ is the electrostatic gating efficiency $\left(\Phi_{\mathrm{QD}}\right.$ is the electrostatic potential at the position of the quantum dot) that determines the change $-\alpha e V$ of the electron's energy in the QD, induced by the applied voltage $V$. Note that, this description is equivalent to Eq. (1) when $E_{0}=0, E_{1}+e^{2} / 2 C_{\Sigma}=\varepsilon_{a}$, $E_{2}+3 e^{2} / 2 C_{\Sigma}=\varepsilon_{b}$, and $C_{s} / C_{\Sigma}=\alpha[7,10,17]$.

When the constant interaction approximation breaks down, the last term in Eq. (1) changes with $N$, and hence, it cannot be neglected. This raises the question regarding the physical meaning of this term. As $C_{t} C_{s} / C_{\Sigma}=$ $\left[\left(1 / C_{s}\right)+\left(1 / C_{t}\right)\right]^{-1}$, this term describes the polarization energy of $C_{s}$ and $C_{t}$, connected in series to the voltage source. Since the inner capacitor plates model the molecular QD [see Figs. 1(a) and 1(b)], a change of this polarization term with $N$ signifies a corresponding change of the molecular polarizability. An $N$-dependent polarizability is indeed expected for small (molecular) QDs. Thus, we develop our model beyond the constant interaction approximation by augmenting it with a molecular polarization energy term. This term will have the generic form $-\frac{1}{2} \gamma(N) \mathcal{E}^{2}$, where $\gamma$ is the molecular polarizability and $\mathcal{E}$ is the electric field at the QD. With $\mathcal{E} \simeq-\left(\Phi_{\mathrm{QD}} / d\right)$ (Ref. [13] shows that the voltage between the tip and the QD drops almost entirely over the bond $d$ ) [21] and $\Phi_{\mathrm{QD}} \simeq\left(d \Phi_{\mathrm{QD}} / d V\right) V$, we obtain

$$
A_{\mathrm{MM}}(V, N)=\varepsilon(N)-N \alpha e V-\frac{1}{2} \gamma(N) \alpha^{2} V^{2} / d^{2}
$$

for the Helmholtz free energy of the molecular QD in the molecular model beyond the constant interaction approximation. The advantage of Eq. (4) is its lucidity, stemming from the fact that it is solely formulated in terms of molecular properties $\varepsilon(N), \gamma(N)$, and $d$. Note, however, that Eq. (4) is mathematically fully equivalent to Eq. (1) if $(\gamma \alpha / d)(1 / d)=C_{\mathrm{t}}$ [22]. We also point out that in Eq. (4), we allow an $N$ dependence of $\gamma$ but keep $\alpha$ constant, which is possible in the framework of the molecular model but inconsistent with the single electron box model in Eq. (1). This reflects an interesting feature of the molecular model, namely that it is not a mere algebraic reformulation of the single electron box model but contains additional, quite subtle, approximations.

Writing the charging condition [Eq. (2)] and the force steps [Eq. (3)] with the newly defined $A_{\mathrm{MM}}(V, N)$ [Eq. (4)] yields

$$
\begin{aligned}
\alpha & =\frac{1}{e\left(V_{+}-V_{-}\right)}\left(\frac{V_{+} \Delta F_{-}}{\partial_{z} V_{-}}+\frac{V_{-} \Delta F_{+}}{\partial_{z} V_{+}}\right), \\
\Delta \gamma & =\frac{d^{2}}{\alpha^{2} V_{-}}\left(\frac{\Delta F_{-}}{\partial_{z} V_{-}}-e \alpha\right)=-\frac{d^{2}}{\alpha^{2} V_{+}}\left(\frac{\Delta F_{+}}{\partial_{z} V_{+}}+e \alpha\right), \\
\varepsilon_{a} & =e \alpha V_{-}+\frac{\Delta \gamma}{2}\left(\frac{\alpha V_{-}}{d}\right)^{2}, \\
\varepsilon_{b} & =e \alpha V_{+}+\frac{\Delta \gamma}{2}\left(\frac{\alpha V_{+}}{d}\right)^{2} .
\end{aligned}
$$

Here, we have set $\gamma(1)-\gamma(0)=\gamma(2)-\gamma(1) \equiv \Delta \gamma$ to obtain a unique solution for $\left(\Delta \gamma, \alpha, \varepsilon_{a}, \varepsilon_{b}\right)$ in terms of measured quantities $\left(V_{ \pm}, \Delta F_{ \pm}, \partial_{z} V_{ \pm}\right)$. Note that the $\partial_{z} V_{ \pm}$ follow from numerical differentiation of $V_{ \pm}(z)$ in Fig. 2(a). For the three molecules studied here, this solution is presented in Fig. 3.

The first observation to notice in Fig. 3 is that except for $\alpha$, all model parameters depend weakly on $z$. This supports the validity of our model Eq. (4), which describes the QD in terms of molecular properties weakly dependent on $z$ [23]. By its definition, $\alpha$ specifies the fraction of the potential difference $V$ that drops over the bond between the QD and the grounded tip that, evidently, must depend on $z$. In fact, the gating efficiency varies slightly from experiment to experiment, reflecting changes in the tip apex curvature (see Supplemental Material [24]). 


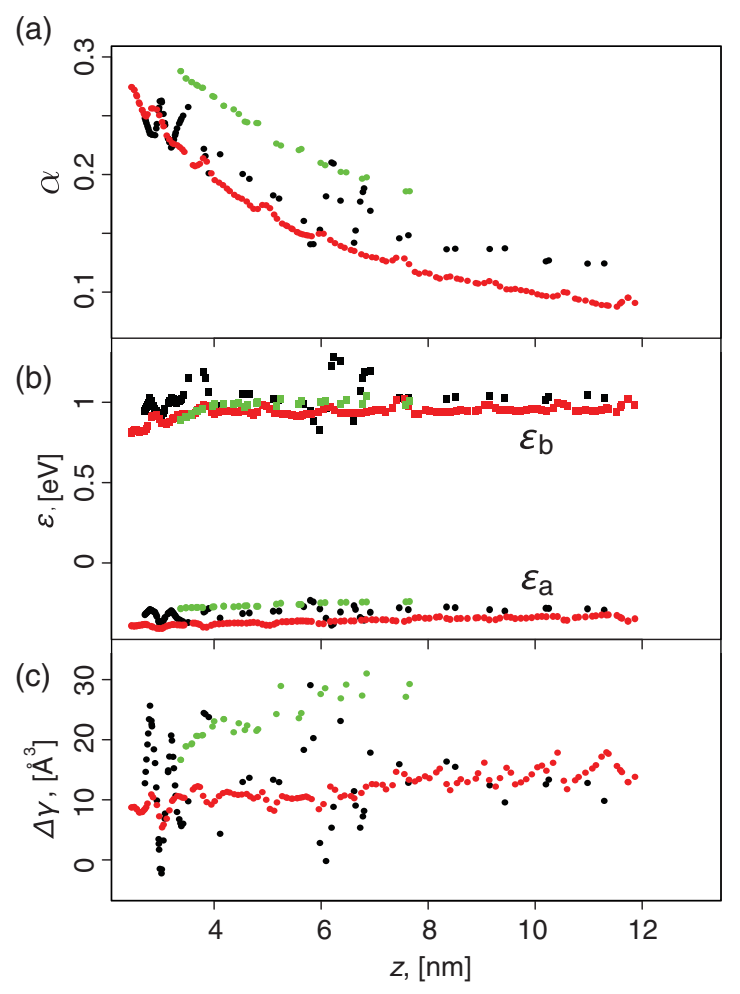

FIG. 3. Parameters of molecular QDs evaluated from the experiment. Color coding as in Fig. 2. (a) Gating efficiency $\alpha$. (b) $\varepsilon_{a}$ (circles) and $\varepsilon_{b}$ (squares). (c) Polarizability change $\Delta \gamma$. The noise in the data arises from the numerical differentiation $\partial_{z} V_{ \pm}$in Eqs. (5)-(8).

We now discuss the molecular (QD) properties determined from the experiment and summarized in Table I. Interestingly, $\varepsilon_{a}$ and $\varepsilon_{b}$ of PTCDA-Cl ${ }_{4}$ are shifted almost uniformly to lower values in comparison to PTCDA. This is consistent with the additional stabilization of the valence $\pi$ orbitals of PTCDA-Cl 4 by four chlorine atoms, and hence, it leads to the assumption that both QD levels may correspond to the same molecular orbital; thus, $\varepsilon_{b}=\varepsilon_{a}+U$, where $U$ is the intraorbital repulsion. Then, $U \simeq 1.3 \mathrm{eV}$ for PTCDA and PTCDA-Cl ${ }_{4}$, which for PTCDA is in excellent agreement with a calculated value of approximately $1.4 \mathrm{eV}$ in the present configuration $[25,26]$ and in line with the expectation that PTCDA-Cl ${ }_{4}$, because of its similar structure and size, has essentially the same $U$. Note that, as expected, the larger TTCDA has a slightly smaller $U$.

The congruence of $U$ values strengthens the hypothesis that $\varepsilon_{a}$ and $\varepsilon_{b}$ correspond to changes in occupancy of one

TABLE I. Experimental values, averaged over $z$, extracted via Eqs. (5)-(8) from the data in Fig. 2. Compare also to Fig. 3.

\begin{tabular}{lcccc}
\hline \hline Molecule & $\varepsilon_{a}(\mathrm{eV})$ & $\varepsilon_{b}(\mathrm{eV})$ & $U(\mathrm{eV})$ & $\Delta \gamma\left(\AA^{3}\right)$ \\
\hline PTCDA & -0.30 & 1.04 & 1.34 & $14 \pm 5$ \\
PTCDA-Cl $_{4}$ & -0.36 & 0.94 & 1.30 & $12 \pm 5$ \\
TTCDA & -0.26 & 1.00 & 1.26 & $25 \pm 5$ \\
\hline \hline
\end{tabular}

molecular orbital only for each of the tested QDs. This orbital can be identified as the LUMO, if we recall that a single PTCDA molecule lifted from $\mathrm{Ag}(111)$ with the STM tip exhibits the Kondo effect due to a single occupancy of its former LUMO [25-27]. In the limit of the upright geometry, which we have here, the Kondo temperature $T_{K}$ drops below $1 \mathrm{~K}$, because the hybridization between the molecule and surface is strongly reduced [25,26], with the result that the system enters the quantum dot regime, with one electron left in the former LUMO, transforming it into a singly occupied molecular orbital (SOMO). This SOMO turns back into a LUMO at $V_{-}$, because the single electron is removed from it. While at $V_{+}$, one electron is added to the SOMO, thus changing it into a new highest occupied molecular orbital (HOMO). Note that the present experiment provides the first quantitative information regarding the tip-suspended molecule, in particular its occupancy, energy level alignment, and intraorbital repulsion.

To compare $\Delta \gamma$ values obtained from our experiments [Fig. 3(c)], we theoretically evaluated the molecular polarizability from the optical transitions of the molecule in the gas phase. These are given by coupled electron-hole excitations (excitons), as resulting from the BetheSalpeter equation within many-body perturbation theory. This approach yields highly reliable excitation energies for molecules and solids $[28,29]$. The results of calculations are shown in Table II. Apart from the fact that the experimental values are systematically larger than the calculated ones, we observe an excellent agreement. In particular, the fact that $\Delta \gamma$ for PTCDA and PTCDA-Cl $l_{4}$ are essentially the same, while the value for TTCDA is twice as large, is predicted correctly by the simulation. The enhancement of the experimental values is in fact not surprising, since it is attributable to the proximity of the highly polarizable metal tip (image dipoles). For a flat electrode, this effect would give an enhancement factor of 2, which serves as an upper limit for the actual enhancement value. Thus, although introduced as molecular polarizability, $\gamma$ is in fact the polarizability of the combined molecule-tip system. Note that, the calculation also confirms the fact that the molecules are indeed oriented with their long axes pointing towards the surface [Fig. 1(b)]. In this configuration, it is the long axis polarizabilities that are relevant, and this is borne out by the comparison between the experiment and calculation. Calculated polarizabilities in the other directions are at least one order of magnitude smaller.

TABLE II. Simulated molecular polarizabilities $\gamma(N)$ (in $\AA^{3}$ ) along the long molecular axis.

\begin{tabular}{lrrcc}
\hline \hline Molecule & $\gamma(0)$ & $\gamma(2)$ & $\Delta \gamma($ theo $)$ & {$[\Delta \gamma(\exp )] /[\Delta \gamma($ theo $)]$} \\
\hline PTCDA & 101 & 115 & 7 & 2 \\
PTCDA-Cl $_{4}$ & 97 & 106 & 5 & 2.4 \\
TTCDA & 206 & 234 & 14 & 1.8 \\
\hline \hline
\end{tabular}


M. R. and F. S. T. are grateful for financial support from the Deutsche Forschungsgemeinschaft (DFG) through SFB 1083 "Structure and Dynamics of Internal Interfaces" (projects A 12 and A 13).

*Corresponding author.

r.temirov@fz-juelich.de

[1] H. Grabert and M. H. Devoret, Single Charge Tunneling (Springer, New York, 1992).

[2] L.P. Kouwenhoven, D. G. Austing, and S. Tarucha, Few-electron quantum dots, Rep. Prog. Phys. 64, 701 (2001).

[3] K. K. Likharev, Single-electron devices and their applications, Proc. IEEE 87, 606 (1999).

[4] M. Macucci, K. Hess, and G. J. Iafrate, Electronic energy spectrum and the concept of capacitance in quantum dots, Phys. Rev. B 48, 17354 (1993).

[5] G. J. Iafrate, K. Hess, J. B. Krieger, and M. Macucci, Capacitive nature of atomic-sized structures, Phys. Rev. B 52, 10737 (1995).

[6] T. LaFave and R. Tsu, Capacitance: A property of nanoscale materials based on spatial symmetry of discrete electrons, Microelectron. J. 39, 617 (2008).

[7] N. Kocić, P. Weiderer, S. Keller, S. Decurtins, S.-X. Liu, and J. Repp, Periodic charging of individual molecules coupled to the motion of an atomic force microscopy tip, Nano Lett. 15, 4406 (2015).

[8] C. Wagner, M. F. B. Green, P. Leinen, T. Deilmann, P. Krüger, M. Rohlfing, R. Temirov, and F. S. Tautz, Scanning Quantum Dot Microscopy, Phys. Rev. Lett. 115, 026101 (2015).

[9] M. F. B. Green, C. Wagner, P. Leinen, T. Deilmann, P. Krüger, M. Rohl, F. S. Tautz, and R. Temirov, Scanning quantum dot microscopy: A quantitative method to measure local electrostatic potential near surfaces, Jpn. J. Appl. Phys. 55, 08 NA04 (2016).

[10] N. Kocić, S. Decurtins, S.-X. Liu, and J. Repp, Forces from periodic charging of adsorbed molecules, J. Chem. Phys. 146, 092327 (2017).

[11] F. J. Giessibl, Advances in atomic force microscopy, Rev. Mod. Phys. 75, 949 (2003).

[12] L. Gross, F. Mohn, P. Liljeroth, J. Repp, F. J. Giessibl, and G. Meyer, Measuring the charge state of an adatom with noncontact atomic force microscopy, Science 324, 1428 (2009).

[13] C. Toher, R. Temirov, A. Greuling, F. Pump, M. Kaczmarski, G. Cuniberti, M. Rohlfing, and F. S. Tautz, Electrical transport through a mechanically gated molecular wire, Phys. Rev. B 83, 155402 (2011).

[14] C. Wagner, N. Fournier, F. S. Tautz, and R. Temirov, Measurement of the Binding Energies of the Organic-Metal Perylene-Teracarboxylic- Dianhydride/Au(111) Bonds by Molecular Manipulation Using an Atomic Force Microscope, Phys. Rev. Lett. 109, 076102 (2012).

[15] C. Wagner, N. Fournier, V. G. Ruiz, C. Li, K. Müllen, M. Rohlfing, A. Tkatchenko, R. Temirov, and F. S. Tautz, Non-additivity of molecule-surface van der Waals potentials from force measurements, Nat. Commun. 5, 5568 (2014).

[16] R. Stomp, Y. Miyahara, S. Schaer, Q. Sun, H. Guo, P. Grutter, S. Studenikin, P. Poole, and A. Sachrajda, Detection of Single-Electron Charging in an Individual InAs Quantum Dot by Noncontact Atomic-Force Microscopy, Phys. Rev. Lett. 94, 056802 (2005).

[17] Y. Miyahara, A. Roy-Gobeil, and P. Grutter, Quantum state readout of individual quantum dots by electrostatic force detection, Nanotechnology 28, 064001 (2017).

[18] M. T. Woodside and P. L. McEuen, Scanned probe imaging of single-electron charge states in nanotube quantum dots, Science 296, 1098 (2002).

[19] M. F. B. Green, Creating and characterizing a single molecule device for quantitative surface science, Dissertation, RWTH, Aachen University, 2018.

[20] A rough estimate of the nonrigidity of the QD confining potential based on the present data shows that at $V_{+}$, the center of the potential moves away from the tip by about $0.1 \pm 0.05 \mathrm{~nm}$ (PTCDA) [19]. This sizable breakdown of orbital rigidity, though not to be taken literally, means that any theoretical description must focus on the effect of electron occupancy on the QD.

[21] The precise value of the electric field depends, of course, on $d$. A larger $d$ than the bond length of $2.2 \AA$, which is a lower bound for the distance over which the voltage drops, will lead to a smaller field.

[22] We note in passing that $(\gamma \alpha / d)$ plays the role of an effective area of the tip capacitor plate, multiplied by a dielectric constant. This finding illustrates the difficulty of defining capacitances in atom-scale systems.

[23] Screening by the metal surface will have a small influence on both $\epsilon_{\mathrm{b}}$ and $U$ and will induce a weak $z$ dependence, which is indeed visible in the data in Fig. 3(b).

[24] See Supplemental Material at http://link.aps.org/ supplemental/10.1103/PhysRevLett.120.206801 for the tip dependence of the experimental measurements.

[25] A. Greuling, M. Rohlfing, R. Temirov, F. S. Tautz, and F. B. Anders, $A b$ initio study of a mechanically gated molecule: From weak to strong correlation, Phys. Rev. B 84, 125413 (2011).

[26] A. Greuling, R. Temirov, B. Lechtenberg, F. B. Anders, M. Rohlfing, and F. S. Tautz, Spectral properties of a molecular wire in the Kondo regime, Phys. Status Solidi B 250, 2386 (2013).

[27] R. Temirov, A. Lassise, F. B. Anders, and F. S. Tautz, Kondo effect by controlled cleavage of a single-molecule contact, Nanotechnology 19, 065401 (2008).

[28] B. Baumeier, M. Rohlfing, and D. Andrienko, Electronic excitations in push-pull oligomers and their complexes with fullerene from many-body Green's functions theory with polarizable embedding, J. Chem. Theory Comput. 10, 3104 (2014).

[29] Y. Ma, M. Rohlfing, and C. Molteni, Excited states of biological chromophores studied using many-body perturbation theory: Effects of resonant-antiresonant coupling and dynamical screening, Phys. Rev. B 80, 241405(R) (2009). 